\title{
CAPÍTULO XXXVII
}

\section{CARACTERIZACIÓN DE ESTILOS DE APRENDIZAJE. ESTUDIO DE CASO: ESTUDIANTES DE LA CORPORACION UNIVERSITARIA MINUTO DE DIOS - UNIMINUTO, CALI, COLOMBIA}

\author{
Jacqueline Neira Parra \\ MEd. En Educación y nuevas tecnologías. Corporación Universitaria del Minuto \\ de Dios, Uniminuto. Correo electrónico: jpjacquie1725@gmail.com. https:// \\ orcid.org/0000-0002-3058-4540.

\section{Héctor Orlando Tarazona Galán} \\ MEd. En Prácticas Pedagógicas, Corporación Universitarita del Minuto de Dios \\ Uniminuto. Correo electrónico: ing.hector_tarazona@yahoo.es. https://orcid. \\ org/0000-0001-7838-7282.
}

\section{Jorge Eliécer Villarreal Fernández}

Lic. Matemáticas y Física, candidato a doctor en Ciencias de la Educación, Corporación Universitaria Americana. Correo electrónico: jvillarealf@ coruniamericana.edu.co. https://orcid.org/0000-0002-4615-8829.

\section{Resumen}

Esta investigación tuvo como objetivo general caracterizar los estilos de aprendizaje de los estudiantes de la Corporación Universitaria Minuto de Dios - Uniminuto (Cali, Colombia) orientados a factores como la edad, el estrato socioeconómico, el semestre matriculado, programa que cursa y el título anterior obtenido. El diseño de la investigación fue deductivo y descriptivo, apoyado en un instrumento de recolección, empleando el cuestionario Honey-Alonso de Estilos de Aprendizaje (CHAEA), agrupando 20 ítems por estilo de aprendizaje, aplicada a 90 estudiantes de cinco programas académicos de la Corporación Universitaria Minuto de Dios. Dentro de los estilos de aprendizaje abordados se encuentran el activo, reflexivo, teórico y pragmático, encontrándose que a medida que se avanza en los semestres se va disminuyendo la variabilidad frente a la preferencia por los estilos de aprendizaje en los programas académicos abordados, siendo el reflexivo el estilo dominante. Los resultados obtenidos permiten dar bases en cuanto al diseño y desarrollo de actividades de aprendizaje significativo, orientadas a potenciar el aprendizaje de los estudiantes desde el inicio del proceso de formación, observando el comportamiento de sus estilos de aprendizaje, buscando favorecer su

www.doi.org/10.47212/tendencias2020vol.xii.38 
rendimiento académico y, además, entregar información importante para replantear estrategias de apoyo al proceso de aprendizaje.

Palabras clave: educación superior, estilos de aprendizaje, estudio descriptivo.

\title{
CHARACTERIZATION OF LEARNING STYLES. CASE STUDY: STUDENTS OF THE CORPORACION UNIVERSITARIA MINUTO DE DIOS - UNIMINUTO, CALI, COLOMBIA
}

\begin{abstract}
This research had the general objective of characterizing the learning styles of the students of the Corporación Universitaria Minuto de Dios - Uniminuto (Cali, Colombia) oriented to factors such as age, socioeconomic status, enrolled semester, current program and previous degree obtained. The research design was deductive and descriptive, supported by a collection instrument, using the Honey-Alonso Learning Styles (CHAEA) questionnaire, grouping 20 items by learning style, applied to 90 students from five academic programs of the Corporación Universitaria Minuto de Dios. Among, the learning styles addressed are active, reflective, theoretical and pragmatic, finding that as one advances in the semesters, the variability decreases compared to the preference for learning styles in the academic programs addressed, being the reflective the dominant style. The results obtained allow us to provide a basis for the design and development of meaningful learning activities, aimed at enhancing student learning from the beginning of the training process, observing the behavior of their learning styles, seeking to promote their academic performance and, in addition, provide important information to rethink strategies to support the learning process.
\end{abstract}

Keywords: education, learning styles, descriptive study.

\section{Proyecto de Investigación}

"Contribución del aprendizaje significativo y los espacios de aprendizaje activo para el desarrollo de competencias profesionales de los estudiantes de Tecnología en Logística Cali", y de la investigación doctoral denominado "Las relaciones entre la motivación, estilos y estrategias de aprendizaje en estudiantes de pregrado de la Corporación Universitaria Americana". 


\section{Introducción}

El enfoque de aprendizaje centrado en el estudiante implica una transformación del paradigma de la enseñanza, convirtiendo al docente en un facilitador, y a su vez, los alumnos toman en sus manos partes esenciales de su proceso, por ejemplo, asumirse como aprendices permanentes (Tarazona y Parra, 2019). Ante esto, el conocimiento del propio proceso de aprendizaje permite conocer la manera en que a alguien le guste aprender o lo que es lo mismo saber cuál es su estilo de aprendizaje y de acuerdo con Barbosa y Pinzón (2018) al identificar estas variables en los estudiantes que ingresan a los procesos de formación superior, se puedan tomar las medidas necesarias para el acompañamiento de sus procesos y determinar las posibles transformaciones que lleven a un mejoramiento de la calidad y evitar así la deserción. El desconocimiento de la manera en que el estudiante aprende, de la forma en que enfrentan los procesos a realizar y la manera en que se procesa la información que se recibe, Ortiz (2013) refiere, que son algunas de las situaciones que se presentan en la educación superior, y no se tienen en cuenta, ni se conocen en muchas ocasiones los elementos que intervienen en ese proceso. En este contexto, es importante conocer, comprender y analizar las condiciones individuales de los estudiantes y los estilos de aprendizaje de los futuros profesionales. Además, es primordial determinar categorías relacionadas con el rendimiento académico, porque muestran que están asociadas con el abandono de la educación y con la baja eficiencia (Cabrera et al, 2006; Cornejo y Redondo, 2007). En los últimos años se han realizado diferentes investigaciones que tienen como centro los estilos de aprendizaje y en los que se ha mostrado que esta categoría, aunque por sí misma es importante, está relacionada con otras o, por lo menos, debe tenerse en cuenta en compañía de otras, como estrategias de aprendizaje, motivación, estrategias de enseñanza, enfoques de aprendizaje, y que buscan el mejoramiento de los resultados académicos de los estudiantes universitarios (Ortiz, 2013). Es por ello, que para dar luz a este respecto la presente investigación busca caracterizar los estilos de aprendizaje de los estudiantes de la Corporación Universitaria Minuto de Dios - Uniminuto, Cali, Colombia. 


\section{Fundamentación teórica}

\section{Estilos de aprendizaje}

Si se comprende el aprendizaje como un proceso individual, que tiene como base las experiencias de cada persona, hay un alejamiento de la generalización que propone el enfoque conductista del aprendizaje (Biggs, 1987). Esto es sustentado en la diversidad de las personas, de las posibilidades, capacidades, habilidades individuales, por lo cual las universidades deberían adecuar los procesos de enseñanza a estas diferencias. Para lograr esto se requiere un reconocimiento de diferentes características propias de los estudiantes, una de ellas los estilos de aprendizaje, el que se logre determinar esto en los alumnos permitirá que se procure una mejor calidad de la educación. En la tabla 1, se pueden observar los diversos modelos que agrupan estilos de aprendizaje, que abarca desde 1970 y los autores que lo plantean (Jürgens, 2016). Alonso et al. (1994, p. 48) explican que "el concepto de Estilo de Aprendizaje no es común para todos los autores", y aportan algunas de las definiciones que se contemplan en la tabla 2.

\section{Tabla 1}

Modelos que agrupan estilos de aprendizaje

\begin{tabular}{|c|c|c|}
\hline Modelo & Fundamento & Autor/Año \\
\hline $\begin{array}{l}\text { Modelos basados en el } \\
\text { proceso de aprendizaje } \\
\text { por experiencia. }\end{array}$ & $\begin{array}{l}\text { Agrupación de acuerdo a la manera } \\
\text { preferida por cada individuo para } \\
\text { integrar y procesar la información, } \\
\text { que permita la solución de } \\
\text { problemas. }\end{array}$ & $\begin{array}{l}\text { Kolb (1976); Honey y } \\
\text { Mumford (1986); Alonso, } \\
\text { Gallego y Honey (1999). }\end{array}$ \\
\hline $\begin{array}{l}\text { Modelos centrados en } \\
\text { la orientación hacia el } \\
\text { estudio. }\end{array}$ & $\begin{array}{l}\text { Su clasificación se relaciona con la } \\
\text { motivación y las actitudes frente al } \\
\text { aprendizaje. }\end{array}$ & $\begin{array}{l}\text { Entwistle (1979); Biggs } \\
(1978-1985) ; \quad \text { Schmeck } \\
(1977) .\end{array}$ \\
\hline $\begin{array}{l}\text { Modelos } r \text { basados } \\
\text { en las preferencias } \\
\text { instruccionales. }\end{array}$ & $\begin{array}{l}\text { Centrados en el componente } \\
\text { perceptual. }\end{array}$ & $\begin{array}{l}\text { Price et al (1976-1977); } \\
\text { Dunn et al (1989); Grasha y } \\
\text { Riechmann (1975). }\end{array}$ \\
\hline $\begin{array}{l}\text { Modelos basados en el } \\
\text { desarrollo de las destrezas } \\
\text { cognitivas. }\end{array}$ & $\begin{array}{l}\text { Centrados en el componente } \\
\text { cognitivo. }\end{array}$ & $\begin{array}{ll}\text { Reinert } & (1976) ; \quad \text { Letteri } \\
(1980) ; & \text { Keefe y Monk } \\
(1986) ; & \text { Keefe (1989-1990). }\end{array}$ \\
\hline
\end{tabular}

Fuente: adaptado de Jürgens (2016). 


\section{Tabla 2}

Definiciones de estilos de aprendizaje

\begin{tabular}{|c|c|c|}
\hline Autor(es) & Año & Definición \\
\hline $\begin{array}{l}\text { Claxton y } \\
\text { Ralston }\end{array}$ & 1978 & $\begin{array}{l}\text { "Estilo de Aprendizaje es una forma consistente de } \\
\text { responder y utilizar los estímulos en un contexto de } \\
\text { aprendizaje." }\end{array}$ \\
\hline Riechmann & 1979 & $\begin{array}{l}\text { "Estilo de Aprendizaje es un conjunto particular de } \\
\text { comportamientos y actitudes relacionados con el } \\
\text { contexto de aprendizaje." }\end{array}$ \\
\hline Butler & 1982 & $\begin{array}{l}\text { "Señalan el significado natural por el que una persona } \\
\text { más fácil, efectiva y eficientemente se comprende a sí } \\
\text { misma, el mundo y la relación entre ambos." }\end{array}$ \\
\hline Kolb & 1984 & $\begin{array}{l}\text { "Algunas capacidades de aprender que se destacan por } \\
\text { encima de otras como resultado del aparato hereditario } \\
\text { de las experiencias vitales propias, y de las exigencias } \\
\text { del medio ambiente actual." "Llegamos a resolver de } \\
\text { manera característica, los conflictos entre el ser activo } \\
\text { y reflexivo y entre el ser inmediato y analítico." }\end{array}$ \\
\hline Keefe & 1988 & $\begin{array}{l}\text { "Los Estilos de Aprendizaje son los rasgos cognitivos, } \\
\text { afectivos y fisiológicos, que sirven como indicadores } \\
\text { relativamente estables, de cómo los discentes } \\
\text { perciben, interaccionan y responden a sus ambientes } \\
\text { aprendizaje". }\end{array}$ \\
\hline
\end{tabular}

Fuente: adaptado de Alonso et al. (1994).

Para esta investigación, y teniendo en cuenta la definición de aprendizaje, se va a tomar como base la definición de Alonso et al. (1994), quienes, partiendo de analizar los modelos anteriores, los definen como los "rasgos cognitivos, afectivos y fisiológicos que sirven como indicadores relativamente estables, de cómo los discentes perciben, interaccionan y responden a sus ambientes de aprendizaje" (p. 48), además realizan un proceso de conceptualización de los estilos de aprendizaje, y plantean etapas para estos, de acuerdo a cada preferencia, se relacionan con cada fase del aprendizaje y son: activo, reflexivo, teórico y pragmático (ver tabla 3). 


\section{Tabla 3}

Fases del proceso de aprendizaje y estilo de aprendizaje relacionado

\begin{tabular}{lccc}
\multicolumn{2}{c}{ Fase del proceso de aprendizaje } & \multicolumn{2}{c}{ Estilo de aprendizaje } \\
\hline Vivir la experiencia & Reflexión & Estilo activo & Estilo reflexivo \\
\hline $\begin{array}{l}\text { Generalización, elaboración } \\
\text { de hipótesis }\end{array}$ & Aplicación & Estilo teórico & Estilo pragmático \\
\hline
\end{tabular}

Fuente: adaptado de Alonso et al. (1994).

Cada estilo de aprendizaje se describe de manera diferente, para Alonso et al. (1994) la cual se puede observar en la tabla 4 .

Las investigaciones de Alonso et al. (1994) prueban que los estudiantes aprenden con mayor efectividad cuando el proceso de enseñanza se enfoca en los estilos de aprendizaje predominantes. El logro académico de los estudiantes universitarios se ve influenciado por factores internos y externos, por tanto, es importante tener presente el papel crucial que juegan las variables personales y contextuales (Monroy y Hernández, 2014). La contextualización de las prácticas pedagógicas a la cotidianidad del estudiante a través de la experimentación, puede convertirse en el mejor aliado del proceso de enseñanza, ya que ellas proporcionan infinidad de posibilidades y oportunidades de aprendizaje, de tipo individual, pero sobre todo colectivo; se convierten también en el mecanismo más directo y eficaz para conocer su realidad, y para aprender de ella (Godínez, et al., 2019). En tal sentido, la investigación planteó la siguiente interrogante ¿De qué manera se asocian las variables estilos de aprendizaje y las características socioeconómicas de los estudiantes objeto de estudio?

\section{Metodología}

El razonamiento que se requiere para realizar esta investigación es deductivo, ya que la categoría, desde la cual se busca caracterizar a la muestra de estudiantes, hacen parte del conocimiento acumulado. Como se reconoce el conocimiento acumulado producto de la historia social, es parte de conocimiento de este (teorías validadas) para generar un nuevo conocimiento, el enfoque es empírico analítico. Igualmente, un estudio observacional, a nivel descriptivo, ya que se buscó caracterizar los Estilos de Aprendizaje sin realizar proceso de intervención. Dado que no se hizo un seguimiento temporal del fenómeno ni se analizaron los cambios en el tiempo de un mismo grupo el 


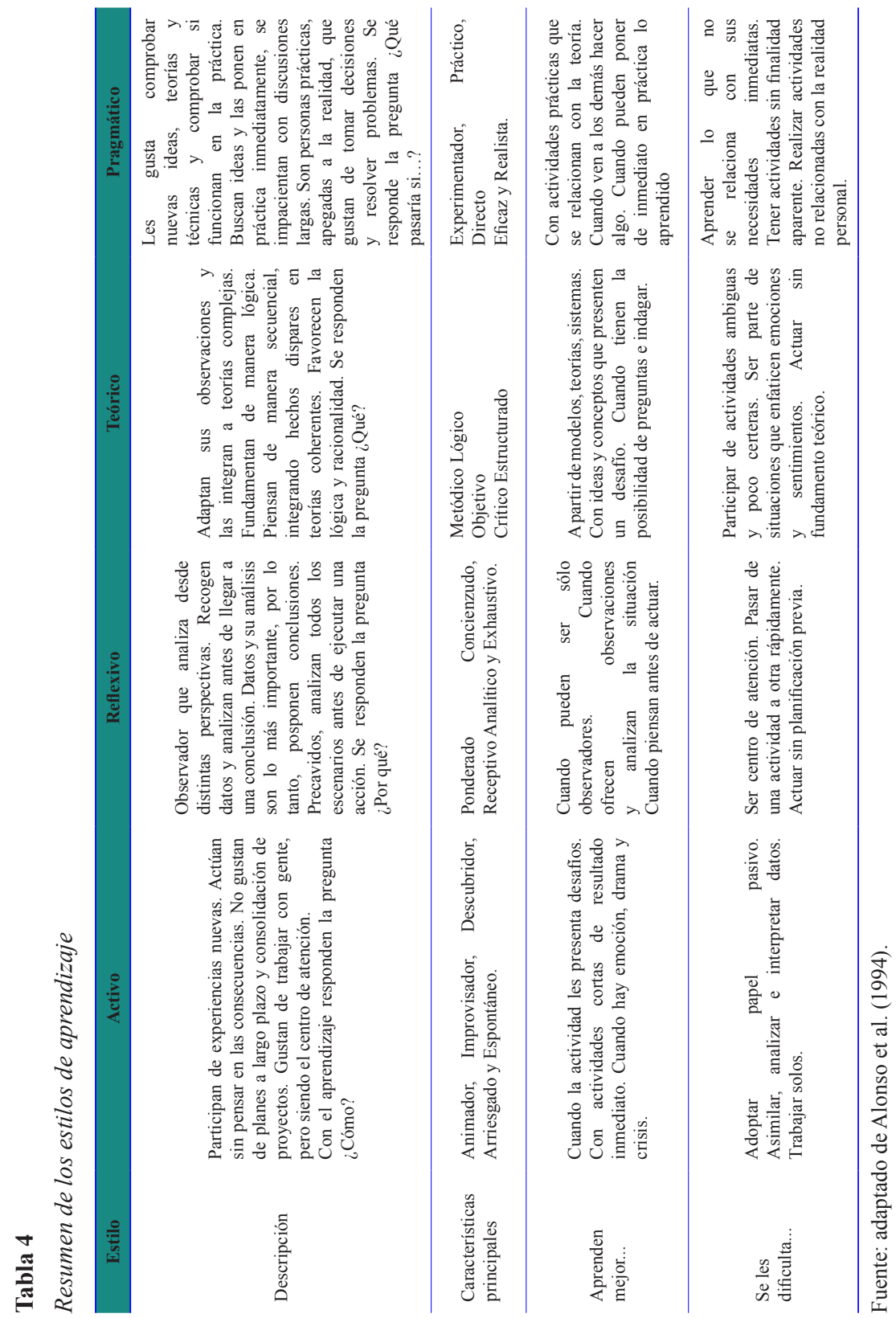


diseño es transversal. La variable analizada en esta investigación es el estilo de aprendizaje. Las variables socio académicas se escogen teniendo en cuenta la diversidad de la población y son el programa que estudian, el género, el semestre que se cursa, si se ha obtenido un título académico o no anteriormente, estrato social, si se tiene una actividad laboral y el tipo de colegio donde se egresó, la edad, los años que lleva en la carrera, si tiene empleo o no, si la institución de donde egresó de la educación media era pública o privada y si ha realizado o no estudios terciarios anteriormente.

Se caracterizaron los estilos de aprendizaje a partir de estas variables socio académicas. La muestra fueron 90 estudiantes de Ingeniería Industrial, Trabajo Social, Tecnología en Logística, Tecnología en Informática y Tecnología en negocios y mercadeo. El muestreo fue a conveniencia, con los estudiantes de los cursos de algunos de los investigadores. El criterio de inclusión fue que quisieran participar y responder el cuestionario, el criterio de exclusión era el no querer participar. Para recolectar los datos se utilizó el cuestionario de auto informe que se envió por medio de encuesta de Google a los correos de los estudiantes, lo cual permite la obtención de información que es esencial para esta investigación. El instrumento para determinar Estilos de Aprendizaje, ha sido validado en varios países. Se ha utilizado en múltiples investigaciones en Colombia y a nivel mundial. Para el caso de esta investigación se revisó para identificar posibles variaciones en el vocabulario y se realizó un proceso de confirmación de validez y confiabilidad. En la tabla 5, se pueden observar las variables e instrumentos de recolección de información.

\section{Tabla 5}

Variables e instrumentos de recolección de información

\begin{tabular}{|c|c|c|}
\hline $\begin{array}{l}\text { Categorías y } \\
\text { características }\end{array}$ & Aspectos medidos & Instrumento \\
\hline $\begin{array}{l}\text { Características socio } \\
\text { académicas }\end{array}$ & $\begin{array}{l}\text { Programa; Género; Edad; Semestre } \\
\text { cursado; Titulación anterior; Estrato } \\
\text { social; Actividad laboral; Tipo de } \\
\text { colegio donde egresó. }\end{array}$ & Cuestionario \\
\hline Estilos de aprendizaje & Pragmático; Teórico; Activo; Reflexivo & $\begin{array}{l}\text { Cuestionario de } \\
\text { Honey-Alonso } \\
\text { de Estilos de } \\
\text { A prendizaje } \\
\text { (CHAEA) }\end{array}$ \\
\hline
\end{tabular}

Fuente: elaboración propia (2020). 


\section{Cuestionario CHAEA para evaluar estilos de aprendizaje}

Hernández et al. (2019) utilizaron el cuestionario Honey-Alonso de Estilos de Aprendizaje (CHAEA) aplicado en una población indígena colombiana, con el propósito de descubrir y caracterizar las particularidades que permitieron articular factores cognitivos, afectivos y fisiológicos característicos, como indicadores de cómo el estudiante percibe, interactúa y responde a su entorno de aprendizaje.

Los estilos de aprendizaje se determinaron a partir del Cuestionario Honey-Alonso de Estilos de Aprendizaje, CHAEA, el cual es una adaptación del cuestionario de Estilos de Aprendizaje LSQ, Learning Styles Questionnaire, (Honey y Mumford, 1986). Tiene 80 ítems, los cuales se organizan en cuatro grupos de 20 ítems, lo que corresponde a cada estilo de aprendizaje (Activo, Reflexivo, Teórico y Pragmático). La respuesta a cada ítem solo puede ser sí o no, se contabilizan las positivas, es necesario que se respondan todas, lo cual se garantizará al incluirlas como obligatorias en un formulario de Google.

La sumatoria de las respuestas positivas permite determinar la preferencia de cada alumno por cada estilo de aprendizaje (García et al., 2009). En cuanto a las normas de interpretación, se debe tener en cuenta la relatividad de las medias obtenidas para cada uno de los estilos de aprendizajes, es decir, que no significa lo mismo obtener la misma media de puntuación en estilo activo que en estilo pragmático. Concretamente el cuestionario CHAEA (Alonso et al., 1994), utiliza un baremo que facilita la interpretación de las puntuaciones obtenidas. Para ello, se agrupan los resultados en cinco niveles, siendo estos:

\section{Tabla 6}

Baremo de interpretación general de preferencia de Estilo de Aprendizaje

\begin{tabular}{ll}
\hline Nivel de preferencia & \multicolumn{1}{c}{ Característica (Representación) } \\
\hline Muy Alta & $10 \%$ de los encuestados con puntuaciones más alta. \\
\hline Alta & $20 \%$ de los encuestados con puntuación Alta. \\
\hline Moderada & $40 \%$ de los encuestados con puntuación nivel medio, es decir entorno a la media. \\
\hline Baja & $20 \%$ de los encuestados con puntuación baja. \\
\hline Muy Baja & $10 \%$ de los encuestados con puntuación más baja. \\
\hline
\end{tabular}

Fuente: Adaptado de Alonso et al. (1994). 
Esta clasificación se puede utilizar como pauta de comparación y orientación para los alumnos de forma individual sobre la importancia de los estilos de aprendizaje, o para orientar a los docentes cuando utilizar un sistema de enseñanza u otro, en el desarrollo de sus clases según los resultados del grupo. La tabla 7, muestra este baremo de interpretación. La prueba de fiabilidad, realizada para cada estilo de aprendizaje dio como resultado de coeficiente Alfa, para el estilo activo 0,680, para el estilo reflexivo, 0,587, para el estilo teórico, 0,518, y para el estilo pragmático, 0,598. La fiabilidad es aceptable considerando la tendencia conservadora de la prueba de Alfa de Cronbach respecto a otras pruebas de fiabilidad.

\section{Tabla 7}

Baremo de interpretación general de preferencia de Estilo de Aprendizaje

\begin{tabular}{lccccc} 
& Muy bajo & Bajo & Moderado & Alto & Muy alto \\
\hline Activo & $0-8$ & $9-10$ & $11-14$ & $15-16$ & $17-20$ \\
\hline Reflexivo & $0-11$ & $12-13$ & $14-17$ & $18-19$ & 20 \\
\hline Teórico & $0-9$ & $10-11$ & $12-15$ & $16-17$ & $18-20$ \\
\hline Pragmático & $0-8$ & $9-10$ & $11-14$ & $15-16$ & $17-20$ \\
\hline
\end{tabular}

Fuente: Alonso et al. (1994).

\section{Resultados}

Frente a la caracterización socioeconómica, en lo que tiene que ver con el sexo, la zona donde se vive, si laboran o no, el tipo de colegio donde se egresó se encuentra en la tabla 8. Otras características, un poco más diversas, se muestra en la tabla 9, donde se consideraron la edad de los participantes que fueron organizados en intervalos, el estrato socioeconómico, el semestre matriculado, el programa que cursa y el título anterior obtenido. Los datos iniciales permiten observar la diversidad de la muestra en cada uno de los ítems a tener en cuenta. Lo menos diverso es la zona donde se vive ya que la mayoría de los estudiantes viven en la zona urbana. 


\section{Tabla 8}

\section{Características socioeconómicas básicas}

\begin{tabular}{cccccccccc} 
& \multicolumn{2}{c}{ Sexo } & Zona vivienda & \multicolumn{2}{c}{$\begin{array}{c}\text { Actividad } \\
\text { laboral }\end{array}$} & \multicolumn{2}{c}{$\begin{array}{c}\text { Tipo de colegio donde } \\
\text { egresó }\end{array}$} \\
\hline $\mathrm{N}=90$ & Masculino & Femenino & Urbana & Rural & Sí & No & Público & Privado \\
\hline Frecuencia & 49 & 41 & 86 & 4 & 49 & 41 & 64 & 26 \\
\hline Porcentaje (\%) & 54,4 & 45,6 & 95,6 & 4,4 & 54,4 & 45,6 & 71,1 & 28,9 \\
\hline
\end{tabular}

Fuente: elaboración propia (2020).

\section{Tabla 9}

\section{Edad de los participantes}

\begin{tabular}{|c|c|c|c|c|c|c|}
\hline \multicolumn{7}{|c|}{ (a). Edad (años) } \\
\hline $\mathbf{N}=90$ & 15 a 20 & 21 a 25 & 26 a 30 & 31 a 35 & 36 a 40 & 41 a 45 \\
\hline Frecuencia & 23 & 28 & 21 & 12 & 5 & 1 \\
\hline Porcentaje $(\%)$ & 25,6 & 31,1 & 23,3 & 13,3 & 5,6 & 1,1 \\
\hline \multicolumn{7}{|c|}{ (b). Estrato Socioeconómico } \\
\hline $\mathrm{N}=90$ & Estrato 1 & Estrato 2 & Estrato 3 & Estrato 4 & Estrato 5 & \\
\hline Frecuencia & 20 & 38 & 29 & 2 & 1 & \\
\hline Porcentaje (\%) & 22,2 & 42,2 & 32,2 & 2,2 & 1,1 & \\
\hline \multicolumn{7}{|c|}{ (c). Semestre matriculado } \\
\hline $\mathbf{N}=\mathbf{9 0}$ & $\begin{array}{c}1^{\mathrm{er}} \\
\text { semestre }\end{array}$ & $\begin{array}{c}2^{\mathrm{do}} \\
\text { semestre }\end{array}$ & $\begin{array}{c}3^{\text {ro }} \\
\text { semestre }\end{array}$ & $4^{\text {to }}$ & $\begin{array}{c}5^{\text {to }} \\
\text { semestre }\end{array}$ & $\begin{array}{c}6^{\text {to }} \\
\text { semestre }\end{array}$ \\
\hline Frecuencia & 25 & 3 & 10 & 23 & 22 & 7 \\
\hline Porcentaje (\%) & 27,8 & 3,3 & 11,1 & 25,6 & 24,4 & 7,8 \\
\hline \multicolumn{7}{|c|}{ (d). Programa que cursan } \\
\hline $\mathbf{N}=90$ & $\begin{array}{l}\text { Ingeniería } \\
\text { Industrial }\end{array}$ & $\begin{array}{c}\text { Tecnología } \\
\text { en } \\
\text { Logística }\end{array}$ & $\begin{array}{c}\text { Trabajo } \\
\text { Social }\end{array}$ & $\begin{array}{c}\text { Tecnología } \\
\text { en } \\
\text { Informática }\end{array}$ & $\begin{array}{l}\text { Tecnología } \\
\text { en negocios } \\
\text { y mercadeo }\end{array}$ & \\
\hline Frecuencia & 19 & 34 & 20 & 14 & 3 & \\
\hline Porcentaje (\%) & 21,1 & 37,8 & 22,2 & 15,6 & 3,3 & \\
\hline \multicolumn{7}{|c|}{ (e). Título anterior al obtenido } \\
\hline $\mathrm{N}=90$ & Ninguno & Técnico & Tecnólogo & Profesional & & \\
\hline Frecuencia & 37 & 41 & 10 & 2 & & \\
\hline Porcentaje (\%) & 41,1 & 45,6 & 11,1 & 2,2 & & \\
\hline
\end{tabular}

Fuente: elaboración propia (2020). 
Algunas características que tienen poca cantidad de personas que en ellas se encuentran, p.e. estudiantes, que ya tienen un título profesional, pueden hacer que los datos no sean tan precisos.

Los resultados de los estilos de aprendizaje se observan en la tabla 10 y junto la figura 1, permiten evidenciar la preferencia de los estudiantes por el estilo de aprendizaje reflexivo, lo cual concuerda con otras investigaciones realizadas del tema en estudiantes universitarios en Colombia (Acevedo y Cavadia, 2015; Madrigal y Trujillo, 2014; Torres et al., 2016). La preferencia más baja es por el estilo de aprendizaje activo y es a la vez el que tiene mayor desviación estándar y amplitud del rango, lo que muestra más dispersión en las respuestas. Teniendo en cuenta el baremo de interpretación los cuatro estilos se encuentran como Moderado, moviéndose en este rango en diferentes intensidades. Igualmente, el figura 1, presenta los resultados de la media para los estilos de aprendizaje. Los valores de frecuencia para cada uno de los baremos de interpretación por estilo de aprendizaje se encuentran en la tabla 11.

Pero la intención de este trabajo es poder caracterizar los estilos no solo en lo general sino particularizando a cada una de las variables socio académicas descritas anteriormente. La caracterización de los estilos de aprendizaje frente al sexo de los participantes se encuentra en la tabla 12 (a) y su comparación con la media general. Las variaciones entre hombres y mujeres y con el puntaje general no varía mucho. Se puede observar una mayor preferencia por los estilos reflexivo y teórico en las mujeres y pragmático en los hombres. Frente al programa estudiado, la figura 2 (a) muestra el puntaje de cada estilo por programa.

Los estudiantes de Ingeniería Industrial tienen las preferencias más bajas en los estilos activo, reflexivo (solo un poco por debajo de Trabajo Social) y teórico. Los estudiantes de Trabajo Social tienen la preferencia más baja por el estilo pragmático. El estilo reflexivo es el más alto para todos los programas. Para esta descripción no se tendrá en cuenta el programa de Tecnología en negocios y mercado por la poca cantidad de estudiantes, solo 3 de los 90 de la muestra. Los estilos de aprendizaje de acuerdo con la edad de los estudiantes se pueden observar en la tabla 12 (b) y sus variaciones en la figura 2 (b). Se puede observar un mayor nivel de variación en el estilo activo con respecto a la edad de los estudiantes. El estilo reflexivo es el más alto para todos los grupos de edad y el activo el más bajo. No se tuvo en cuenta el grupo de 41 a 45 años por solo tener una persona. 
Para caracterizar los estilos de aprendizaje de acuerdo con el semestre matriculado se tomaron en cuenta aquellos semestres donde se tomó como muestra más del $10 \%$ del total. La tabla 12 (c) y la figura 2 (c) muestran los resultados de este cálculo. A medida que se avanza en los semestres se va disminuyendo la variabilidad frente a la preferencia por los estilos de aprendizaje, en el semestre 5 hay menos variabilidad que en los anteriores. También se puede observar cómo el estilo reflexivo permanece más constante durante el transcurrir de los semestres.

Tabla 10

Valor medio estilos de aprendizaje y estadísticos básicos

\begin{tabular}{lcccc} 
Estilo Activo & $\begin{array}{c}\text { Estilo } \\
\text { Reflexivo }\end{array}$ & $\begin{array}{c}\text { Estilo } \\
\text { Teórico }\end{array}$ & $\begin{array}{c}\text { Estilo } \\
\text { Pragmático }\end{array}$ \\
\hline Media & 12,0 & 15,6 & 14,5 & 14,2 \\
\hline Mediana & 12 & 16 & 15 & 14 \\
\hline Moda & 10 & 15 & 16 & 15 \\
\hline Desviación estándar & 3,2 & 2,6 & 2,6 & 2,8 \\
\hline Rango & 16 & 14 & 14 & 12 \\
\hline
\end{tabular}

Fuente: elaboración propia (2020).

\section{Figura 1}

Valores promedio de estilos de aprendizaje de los estudiantes

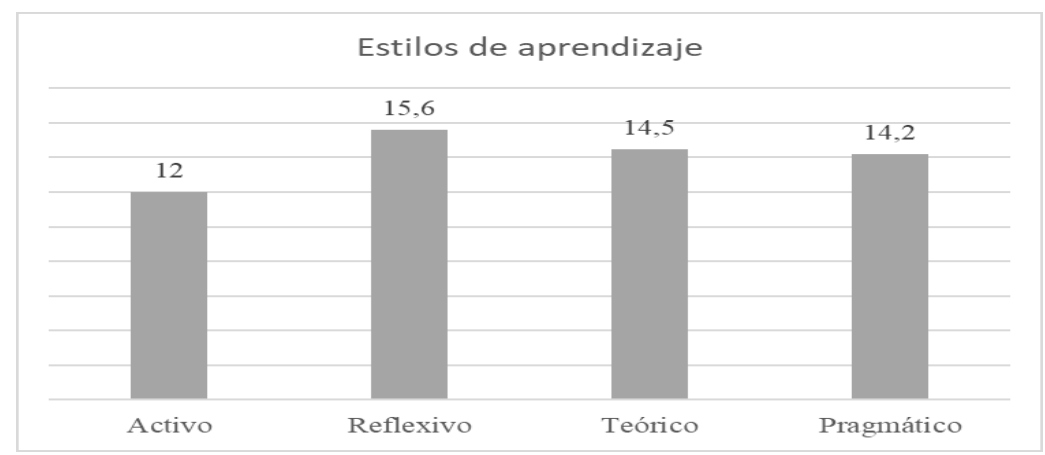

Fuente: Elaboración propia (2020). 


\section{Tabla 11}

Baremos de interpretación para los estilos de aprendizaje

\begin{tabular}{lcccccc}
\multirow{2}{*}{ Estilo } & $\mathbf{N}=\mathbf{9 0}$ & $\begin{array}{c}\text { Muy } \\
\text { bajo }\end{array}$ & Bajo & Moderado & Alto & $\begin{array}{c}\text { Muy } \\
\text { alto }\end{array}$ \\
\hline \multirow{2}{*}{ Activo } & Frecuencia & 14 & 17 & 41 & 11 & 7 \\
\cline { 2 - 7 } & Porcentaje (\%) & 15,6 & 18,9 & 45,6 & 12,2 & 7,8 \\
\hline \multirow{2}{*}{ Reflexivo } & Frecuencia & 2 & 2 & 21 & 30 & 35 \\
\cline { 2 - 7 } & Porcentaje (\%) & 2,2 & 2,2 & 23,3 & 33,3 & 38,9 \\
\hline \multirow{2}{*}{ Teórico } & Frecuencia & 1 & 6 & 34 & 29 & 20 \\
\hline \multirow{2}{*}{ Pragmático } & Porcentaje (\%) & 1,1 & 6,7 & 37,8 & 32,2 & 22,2 \\
\cline { 2 - 7 } & Frecuencia & 1 & 8 & 41 & 21 & 19 \\
\cline { 2 - 7 } & Porcentaje (\%) & 1,1 & 8,9 & 45,6 & 23,3 & 21,1 \\
\hline
\end{tabular}

Fuente: elaboración propia (2020).

La tabla 12 (d) muestra que los estudiantes que han obtenido un título de técnicos tienen un menor nivel de variabilidad de sus estilos de aprendizaje, y su variación se puede observar el gráfico 2 (d). Para el caso de los estilos se puede observar que el estilo reflexivo se mantiene con menor variabilidad, es decir es más constante, tengan o no un título anterior.

La tabla 12 (e) y el gráfico 2 (e), permiten observar las características de acuerdo con el estrato socioeconómico de los estudiantes. Solo se toman en cuenta las variables que cuentan con más del 10\% del total de la muestra, para este caso solo se tomará el estrato 1, 2 y 3 . En ellos, se puede observar que el estilo activo es el preferente para los estudiantes provenientes del estrato $1 \mathrm{y}$ va disminuyendo esta preferencia al aumentar el estrato. El estilo reflexivo sigue siendo el de menos variabilidad y mayor preferencia.

Si se tiene en cuenta el aspecto laboral, la tabla 12 (f) y el gráfico 2 (f) muestran las características de los estilos de aprendizaje a partir de ese aspecto. La menor variación se presenta en las personas que no laboran, mientras que, diferente a lo que ha pasado en los casos anteriores, el estilo que se mantiene más constante es el activo, aunque sigue siendo el de más baja preferencia. El estilo reflexivo sigue siendo el de mayor preferencia. 
La última variable socioeconómica que se tuvo en cuenta en esta investigación fue el tipo de colegio de donde egresó el estudiante, manejándose dos alternativas, público o privado, como se observan en la tabla 12 (g) y la figura 2 (g). La preferencia más alta está en aquellos estudiantes que egresaron de colegio privado, manteniéndose, en ambos casos, la prevalencia del reflexivo y más baja la del activo. Y aunque la diferencia es poca, el estilo activo es menos variable que los demás.

Tabla 12

Estilos de aprendizaje y género de los participantes

\begin{tabular}{|c|c|c|c|c|c|}
\hline \multicolumn{2}{|c|}{$\mathrm{N}=90$} & Activo & Reflexivo & Teórico & Pragmático \\
\hline \multirow{3}{*}{$\begin{array}{l}\text { (12.a) Género de } \\
\text { los participantes }\end{array}$} & Mujeres & 12,0 & 15,8 & 14,8 & 13,9 \\
\hline & Hombres & 12,0 & 15,4 & 14,3 & 14,4 \\
\hline & General & 12 & 15,6 & 15 & 14,2 \\
\hline \multicolumn{2}{|c|}{$\mathrm{N}=90$} & Activo & Reflexivo & Teórico & Pragmático \\
\hline \multirow{5}{*}{$\begin{array}{l}\text { (12.b). Edad } \\
\text { (años) }\end{array}$} & $\begin{array}{l}\text { Entre } 15 \text { y } 20 \\
\text { años }\end{array}$ & 12,3 & 15,0 & 13,9 & 13,5 \\
\hline & $\begin{array}{l}\text { Entre } 21 \text { y } 25 \\
\text { años }\end{array}$ & 12,4 & $.16,1$ & 15,0 & 14,6 \\
\hline & $\begin{array}{l}\text { Entre } 26 \text { y } 30 \\
\text { años }\end{array}$ & 11,6 & 15,5 & 15,0 & 14,8 \\
\hline & $\begin{array}{l}\text { Entre } 31 \text { y } 35 \\
\text { años }\end{array}$ & 10,3 & 15,3 & 13,4 & 13,1 \\
\hline & $\begin{array}{l}\text { Entre } 36 \text { y } 40 \\
\text { años }\end{array}$ & 13,2 & 15,4 & 15,4 & 14,4 \\
\hline \multicolumn{2}{|c|}{$\mathrm{N}=90$} & Activo & Reflexivo & Teórico & Pragmático \\
\hline \multirow{3}{*}{$\begin{array}{l}\text { (12.c). Estrato } \\
\text { Socioeconómico }\end{array}$} & Estrato 1 & 13,3 & 15,4 & 14,9 & 14,1 \\
\hline & Estrato 2 & 11,7 & 15,4 & 14,3 & 13,7 \\
\hline & Estrato 3 & 11,3 & 16,0 & 14,5 & 14,6 \\
\hline \multicolumn{2}{|c|}{$\mathrm{N}=90$} & Activo & Reflexivo & Teórico & Pragmático \\
\hline \multirow{4}{*}{$\begin{array}{l}\text { (12.d). Semestre } \\
\text { matriculado }\end{array}$} & Semestre 1 & 10,8 & 15,5 & 13,8 & 12,9 \\
\hline & Semestre 3 & 11,8 & 15,8 & 14,8 & 14,3 \\
\hline & Semestre 4 & 11,7 & 16,0 & 14,7 & 15,0 \\
\hline & Semestre 5 & 13,0 & 15,5 & 14,8 & 14,7 \\
\hline
\end{tabular}

Fuente: elaboración propia (2020). 
Tabla 12

Estilos de aprendizaje y género de los participantes (continuación)

\begin{tabular}{|c|c|c|c|c|c|}
\hline \multicolumn{2}{|c|}{$\mathrm{N}=90$} & Activo & Reflexivo & Teórico & Pragmático \\
\hline \multicolumn{2}{|c|}{$\mathrm{N}=90$} & Activo & Reflexivo & Teórico & Pragmático \\
\hline \multirow{3}{*}{$\begin{array}{l}\text { (12.e). Título } \\
\text { anterior al } \\
\text { obtenido }\end{array}$} & Ninguno & 11,9 & 15,5 & 14,1 & 13,8 \\
\hline & Técnico & 12,7 & 15,7 & 14,9 & 14,5 \\
\hline & Tecnólogo & 9,3 & 15,1 & 14,9 & 13,5 \\
\hline \multicolumn{2}{|c|}{$\mathrm{N}=90$} & Activo & Reflexivo & Teórico & Pragmático \\
\hline \multirow{2}{*}{$\begin{array}{l}\text { (12.f) Situación } \\
\text { laboral }\end{array}$} & Si labora & 11,8 & 16,1 & 14,9 & 14,7 \\
\hline & No labora & 12,2 & 15,0 & 14,1 & 13,5 \\
\hline \multicolumn{2}{|c|}{$\mathrm{N}=90$} & Activo & Reflexivo & Teórico & Pragmático \\
\hline \multirow{2}{*}{$\begin{array}{l}\text { (12.g) Colegio de } \\
\text { egresó }\end{array}$} & $\begin{array}{l}\text { Colegio } \\
\text { público }\end{array}$ & 11,9 & 15,4 & 14,4 & 14,0 \\
\hline & $\begin{array}{l}\text { Colegio } \\
\text { privado }\end{array}$ & 12,2 & 16,0 & 15,0 & 14,6 \\
\hline
\end{tabular}

Fuente: elaboración propia (2020).

\section{Figura 2}

Estilos de aprendizaje de acuerdo a cada programa académico

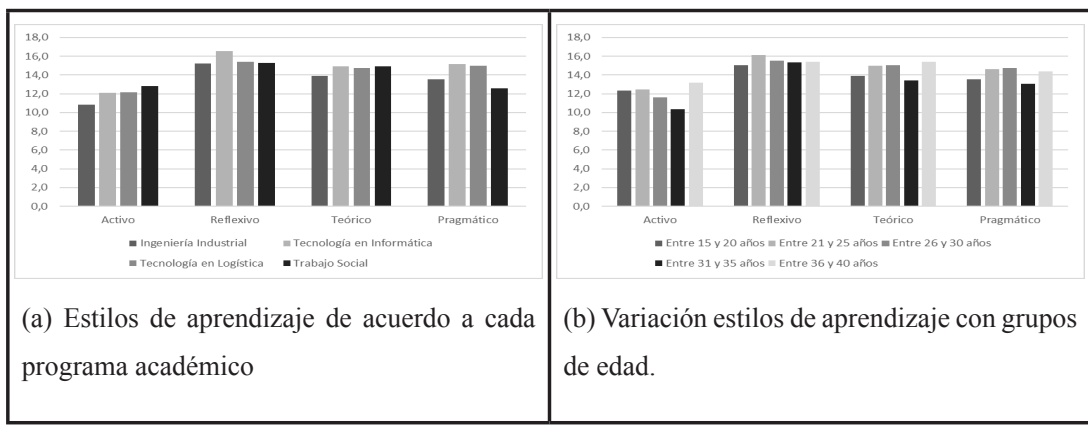

Fuente: elaboración propia (2020). 
Figura 2

Estilos de aprendizaje de acuerdo a cada programa académico

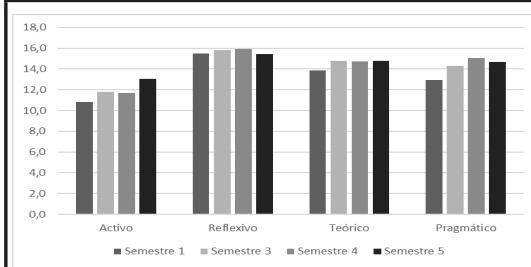

(c) Estilo de aprendizaje por semestre

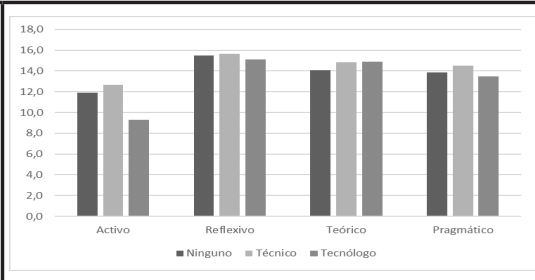

(d) Estilos de aprendizaje por título

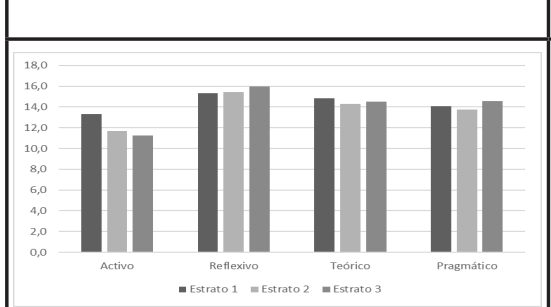

(e) Variación estilos de aprendizaje por estrato socioeconómico

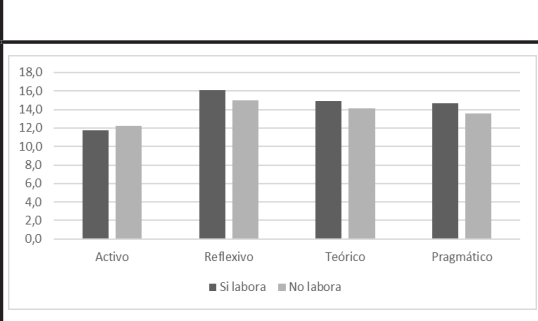

(f) Variación estilos de aprendizaje y situación laboral

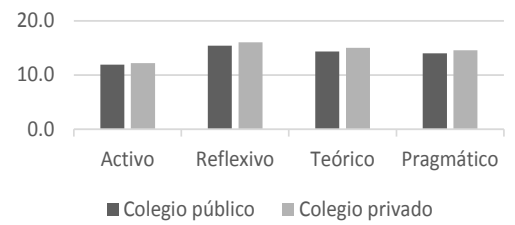

(g) Variación estilos de aprendizaje y tipo de colegio de egreso del estudiante.

Fuente: elaboración propia (2020).

\section{Conclusiones}

Los resultados del proceso investigativo evidencian que, en la muestra de estudiantes seleccionados, el estilo de aprendizaje preferente es el reflexivo, el cual se mantiene en esta condición, aunque se analice cualquiera de las variables socio académicas es 
este estudio. También se pueden observar variaciones en la preferencia de los estilos de aprendizaje que pueden ser aprovechadas en el caso de que se identifiquen y se realicen transformaciones en los estilos de enseñanza acordes con lo hallado. Por lo tanto, es necesario que se pueda indagar sobre los estilos de enseñanza de los docentes que están a cargo de los procesos de enseñanza de estos estudiantes. Esta investigación permite pensar en hipótesis a probar en futuras pesquisas, por lo que es un punto de entrada para profundizar en el nivel de análisis. Se podría analizar si las variaciones encontradas corresponden a relaciones significativas entre variables, para luego llegar, sobre la base de experimentación, a identificar relaciones de causalidad entre ellas. Es necesario ampliar la muestra en cada uno de los programas, de manera que el número aún más significativo y se pueda determinar si es posible realizar inferencias a partir de esos datos encontrados, a la vez realizar comparaciones con estudiantes de la misma universidad, pero de otras regiones, o de otras universidades en los mismos programas, buscando determinar si los estilos son una causa o una consecuencia. Con esta muestra se debe buscar si hay relación entre los estilos de aprendizaje y el rendimiento académico que han tenido los estudiantes en su proceso de aprendizaje, de manera que también se tenga una herramienta de análisis del proceso objetivo del sistema educativo, el aprendizaje de los estudiantes. Los resultados obtenidos podrían dar bases en cuanto al diseño y desarrollo de actividades de aprendizaje significativo orientadas a potenciar el aprendizaje de los estudiantes desde el inicio del proceso de formación y observar a través del avance en el tiempo como se comportan o se transforman sus estilos de aprendizaje.

\section{Referencias bibligráficas}

Acevedo, D. y Cavadia, S. (2015). Estilos de Aprendizaje de los Estudiantes de la Facultad de Ingeniería de la Universidad de Cartagena, Colombia. Revista Estilos de Aprendizaje, 8(4), 15-22. https://doi.org/10.4067/S071850062015000400003

Alonso, C., Gallego, D. y Honey, P. (1994). Procedimientos de diagnóstico y mejora. Madrid: Ediciones Mensajero.

Barbosa, I. y Pinzón, D. (2018). Incidencia de la Financiación en la Deserción Universitaria en Bogotá Ingrid. Universidad Católica de Colombia. 
Biggs, J. (1987). Student Approaches to Learning and Studying. Frederick St: ustralian Council for Educational Research Ltd.

Cabrera, L., Bethencourt, J., Alvarez, P. y González, M. (2006). El problema del abandono de los estudios universitarios. Relieve, 12(2), 171-203.

Cornejo, R, y Redondo, J. (2007). Variables y factores asociados al aprendizaje escolar: una discusión desde la investigación actual. Estudios Pedagógicos (Valdivia), 33(2), 155-175.

García, J., Santizo, J. \& Alonso, C. (2009). Instrumentos de medición de estilos de aprendizaje. Revista de Estilos de Aprendizaje, 4(4), 1-20.

Godínez, R., Ramírez, C., Galindo, M. y Luna, J. (2019). La mejora de los aprendizajes en ciencias III de nivel secundaria a través de la experimentación con materiales de uso común en el salón de clase. En Tendencias en la Investigación Universitaria. Una visión desde Latinoamérica (pp. 188197). Editorial Artes y Letras S.A.S.

Hernández, de J., Vega, A. y Barbera, N. (2019). Diagnóstico de la dominancia cerebral como estrategia para la conformación de equipos de investigación. En Tendencias en la Investigación Universitaria. Una visión desde Latinoamérica (pp. 150-160). Editorial Artes y Letras S.A.S.

Jürgens Schneeberger, K. (2016). Evaluación de la motivación, estilos y estrategias de aprendizaje en estudiantes de la Facultad de Ciencias de la Universidad Austral de Chile. Tesis Doctoral, 456.

Madrigal, A. y Trujillo, J. (2014). Adaptación del cuestionario Honey-Alonso de estilos de aprendizaje para estudiantes de una institución universitaria de MedellínColombia. Revista de Estilos de Aprendizaje, 7(13), 155-181. 
Monroy, F. y Hernández Pina, F. (2014). Factores que influyen en los enfoques de aprendizaje universitario. Una revisión sistemática. Educacion XX1, 17(2), 105-124. https://doi.org/10.5944/educxx1.17.2.11481

Ortiz, A. (2013). Modelos Pedagógicos y Teorías del Aprendizaje. Ediciones de la U.

Tarazona, H. y Parra, N. (2019). La innovación didáctica y la pedagogía activa en el desarrollo de las competencias profesionales de los estudiantes de Tecnología en Logística de Uniminuto sede Cali. In Compilado de Experiencias e Investigaciones en Innovación Educativa DOKUMA 2019 (Vol. 3, pp. 102113). Bogotá,Colombia: Dokuma: Creatividad y Tecnología SAS.

Torres, C., Bolaños, D., de la Peña, L., Hernández, L. y Obando, K. (2016). Identificación de los estilos de aprendizaje de estudiantes de cinco facultades de medicina de Cali, Colombia. In Journal of Learning Styles (Vol. 11). 